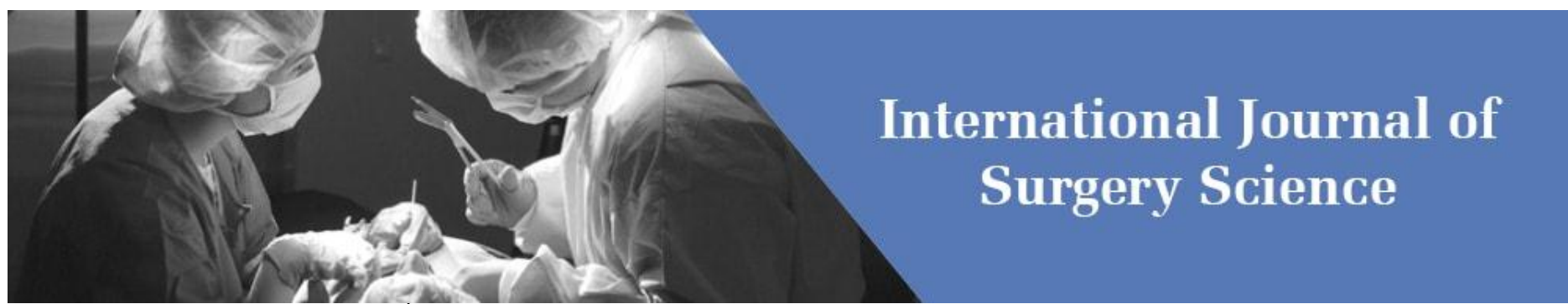

E-ISSN: 2616-3470

P-ISSN: 2616-3462

(C) Surgery Science

www.surgeryscience.com

2022; 6(1): 168-172

Received: 24-11-2021

Accepted: 26-12-2021

Augustin Kibonge Mukakala

a) Department of Surgery, University

Clinics of Bukavu, Faculty of Medicine,

Official University of Bukavu, Bukavu,

DR Congo

b) Department of Surgery, University

Clinics of Lubumbashi, Faculty of

Medicine, University of Lubumbashi,

Lubumbashi, Haut-Katanga, DR Congo

Manix Banza Ilunga

Department of Surgery, University

Clinics of Lubumbashi, Faculty of

Medicine, University of Lubumbashi,

Lubumbashi, Haut-Katanga, DR Congo

Nasser Amisi Lubosha

Department of Surgery, University

Clinics of Lubumbashi, Faculty of

Medicine, University of Lubumbashi,

Lubumbashi, Haut-Katanga, DR Congo

Dimitri Kanyanda Nafatalewa

Department of Surgery, University

Clinics of Lubumbashi, Faculty of

Medicine, University of Lubumbashi,

Lubumbashi, Haut-Katanga, DR Congo

Eric Mbuya Musapudi

Department of Surgery, University

Clinics of Lubumbashi, Faculty of

Medicine, University of Lubumbashi,

Lubumbashi, Haut-Katanga, DR Congo

Tresor Kibangula Kasanga

Department of Surgery, University

Clinics of Lubumbashi, Faculty of

Medicine, University of Lubumbashi,

Lubumbashi, Haut-Katanga, DR Congo

Serge Ngoie yumba

Department of Surgery, University

Clinics of Lubumbashi, Faculty of

Medicine, University of Lubumbashi,

Lubumbashi, Haut-Katanga, DR Congo

Willy Arung Kalau

Department of Surgery, University

Clinics of Lubumbashi, Faculty of

Medicine, University of Lubumbashi,

Lubumbashi, Haut-Katanga, DR Congo

Corresponding Author:

Augustin Kibonge Mukakala

a) Department of Surgery, University

Clinics of Bukavu, Faculty of Medicine,

Official University of Bukavu, Bukavu,

DR Congo

b) Department of Surgery, University

Clinics of Lubumbashi, Faculty of

Medicine, University of Lubumbashi,

Lubumbashi, Haut-Katanga, DR Congo

\section{Rupture of the jejunum by closed trauma of the abdomen: About two cases at the university clinics of Lubumbashi}

\author{
Augustin Kibonge Mukakala, Manix Banza Ilunga, Nasser Amisi Lubosha, \\ Dimitri Kanyanda Nafatalewa, Eric Mbuya Musapudi, Tresor Kibangula \\ Kasanga, Serge Ngoie yumba and Willy Arung Kalau
}

DOI: https://doi.org/10.33545/surgery.2022.v6.i1c.849

\section{Abstract}

Rupture of the small intestine by blunt abdominal trauma is a rare phenomenon and is most often secondary to violent contusions.

The diagnosis is not easy but should be suspected in the face of intense abdominal pain and peritoneal signs (defense evolving towards contracture), after a high-energy trauma.

The authors report two cases of jejunal rupture consecutive to road traffic accidents diagnosed late and followed up at university clinics in Lubumbashi.

Keywords: Jejunal rupture, trauma, abdomen

\section{Introduction}

Introduction Closed abdominal trauma, also called abdominal contusion, is injury produced in the abdomen, its contents or its wall, by a trauma that has respected parietal continuity. They can occur in isolation (sports accident, assault), or more frequently, in the context of polytrauma (public highway accident). They constitute 70 to $86 \%$ of abdominal trauma. Their mortality rate is around $20 \%$, linked to the severity of the single lesions on the one hand, and on the other hand, to a context of polytrauma ${ }^{[1]}$. Rupture of the jejunum by blunt abdominal trauma is exceptional and most often secondary to violent contusions. It is the subject of regular studies and its frequency is low ( 1 to $2 \%$ or even $5 \%$ ) compared to other post-traumatic visceral lesions (liver, spleen in particular) ${ }^{[2]}$. Their diagnostic difficulty is linked to a poor clinical semiology. Management is surgical and requires early diagnosis, as the lesions are potentially serious or even fatal ${ }^{[3]}$. If the therapeutic attitude proves to be well codified, the difficulty of an early diagnosis remains relevant in view of the clinical polymorphism and despite the contribution of new diagnostic techniques, in particular imaging ${ }^{[4,5]}$.

The purpose of this work was to report these two observations and to specify the management of these patients who had a triple particularity: etiological, clinical and evolutionary.

\section{Patients and Observing}

\section{Observation 1:}

A 36-year-old subject, taxi-bus driver, was admitted to the emergency room for abdominal pain dating back 24 hours from the consultation, after a road traffic accident during which, driving at high speed and without a seat belt, he was thrown against the steering wheel of his vehicle during sudden braking. The post-traumatic consequences were marked by abdominal pain for which he had been taken to a nearby health center where he received analgesics and antibiotics of unknown nature and dose before he was transferred to university clinics of Lubumbashi for the best support. He did not report any particular medical-surgical history and his last meal dated back to 6 hours from the consultation. The objective examination reveals a suffering face with a stable hemodynamic state, and a Glasgow score of $15 / 15$. The temperature was $37.8^{\circ} \mathrm{C}$. Her abdomen was bloated, breathing shallowly with no spontaneously visible peristaltic waves. A generalized defense was noted with an umbilical cry present and generalized dullness, hypertympanism in the epigastrium with disappearance of the pre-hepatic dullness. 
The peristaltic noise was weakly perceived. The abdominal washing puncture had brought back a greenish liquid and the pelvic touch had revealed an insensitive bulging Douglas fir. The diagnosis of abdominal contusion with suspicion of a perforation of a hollow organ had been evoked, and an X-ray of the abdomen without preparation made in emergency had shown two gas crescents: one in inter hepato-diaphragmatic and the other in interspleno-diaphragmatic (fig. 1).

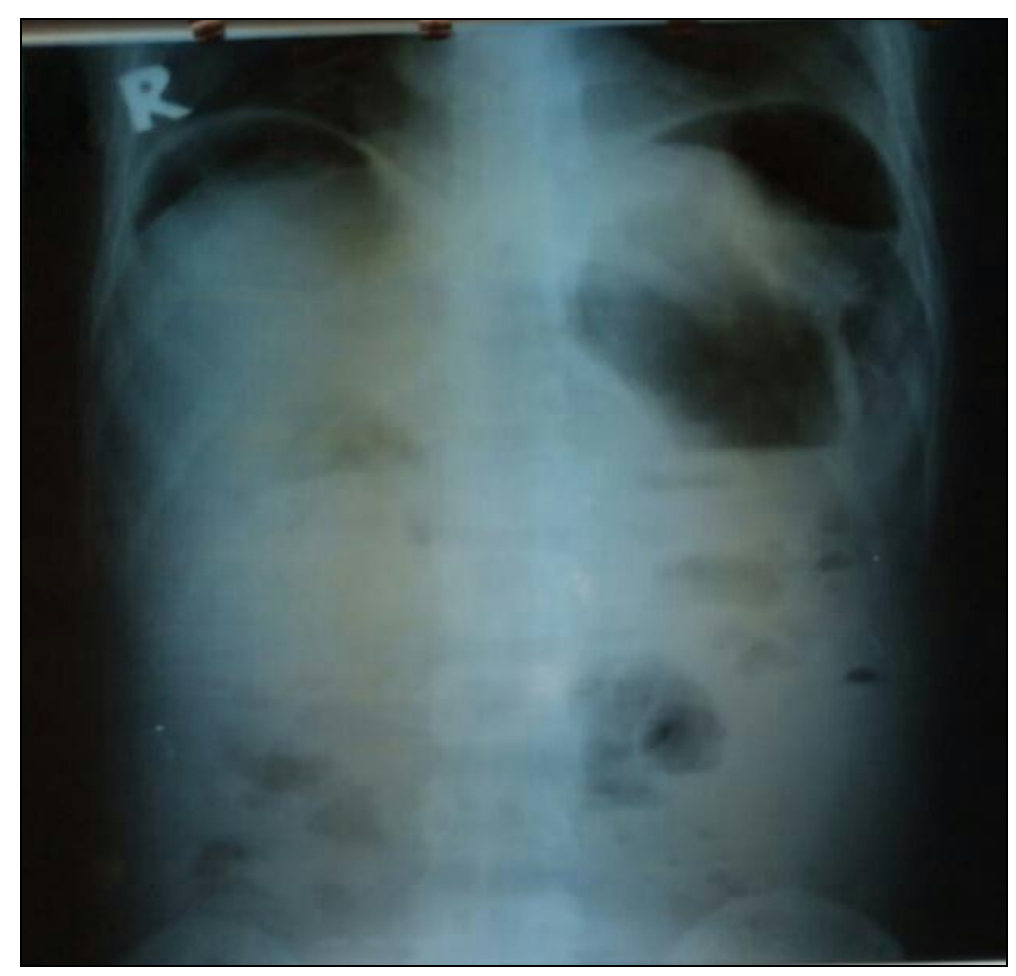

Fig 1: An x-ray of the abdomen without preparation showing two gas crescents: inter hepato-diaphragmatic, and inter gastro-diaphragmatic.

The patient was classified as ASA II and resuscitation measures were instituted. An exploratory laparotomy was performed 24 hours after admission and revealed: A gas and liquid issue, liquid of approximately $2000 \mathrm{ml}$, greenish in appearance, with a fecaloid odor mixed with coagulated blood, a complete section of the jejunum about $120 \mathrm{~cm}$ from the angle of TREITZ, with blunt edges. The surgery consisted of:

- Abundant cleansing of the abdominal cavity with 5 liters of lukewarm $0.9 \% \mathrm{Nacl}$;

- Brightening of the intestinal edges followed by an end-toend anastomosis with silk $2 / 0$ in a plane reinforced by seroserous separate stitches;

- Closure of the abdominal cavity layer by layer, after placement of two lamellar drains in the two colo-parietal gutters, drains removed 72 hours later. The patient had been put under; ceftriaxone $3 \times 1 \mathrm{gr} / \mathrm{d}$ IVD, Metronidazole $3 \times 500 \mathrm{mg} / \mathrm{d}$ IVL, Paracetamol 3x1gr/d IVD, Cimetidine $2 \times 200 \mathrm{mg} / \mathrm{d}$ IVD.

The evolution was marked by the occurrence on postoperative day 7 of a low-flow enteral fistula which was treated medically: lifestyle and dietary measures, slowing of transit with belladonna tincture. The fistula dried up thirteen days later. The patient was discharged from the hospital on postoperative day 22 cured and seen again at the appointment one month later, without any particularities. Observation 2: A 34-year-old male subject consulted in the emergency room for abdominal pain dating back six days, following a road traffic accident during which he was seated in a public transport bus, in the driver's back seat, without a seat belt. He had hit his abdomen against the driver's seat when the bus collided with another which was traveling at high speed. The post-traumatic suites were marked by more marked abdominal pain in the umbilicus, of moderate intensity for which he had self-medicated with Paracetamol Co 500mg, 2x1gr/d//4d, Ibuprofen Co 400mg, 2x400mg/d // 5 days per os without disappearance of the signs. The exacerbation of this pain, the occurrence of postprandial vomiting, and diarrhea motivate consultation at the University Clinics of Lubumbashi for treatment.

\section{Observation 2}

A 34-year-old male subject consulted in the emergency room for abdominal pain dating back six days, following a road traffic accident during which he was seated in a public transport bus, in the driver's back seat, without a seat belt. He had hit his abdomen against the driver's seat when the bus collided with another which was traveling at high speed. The post-traumatic suites were marked by more marked abdominal pain in the umbilicus, of moderate intensity for which he had selfmedicated with Paracetamol Co 500mg, 2x1 gr/d//4d, Ibuprofen Co $400 \mathrm{mg}, 2 \times 400 \mathrm{mg} / \mathrm{d} / / 5$ days per os without disappearance of the signs. The exacerbation of this pain, the occurrence of postprandial vomiting, and diarrhea motivate consultation at the University Clinics of Lubumbashi for treatment. The clinical examination revealed a general condition marked by a fever of $38.8^{\circ} \mathrm{C}$ and peritoneal facies. Tachypnea, thready pulse, tachycardia at $120 \mathrm{btt} / \mathrm{min}$, dry tongue and presence of dark circles were noted. The abdomen was slightly distended, contracted as a whole with an umbilical cry and a sepulchral silence on auscultation. On pelvic touch, Douglas's cul-de-sac was bulging and tender.

A diagnosis of acute surgical abdomen complicated by severe dehydration was suggested, and an emergency radiograph of the abdomen without preparation (ASP) showed an interhepatodiaphragmatic gas crescent with dullness. The patient was classified as ASA III and resuscitation measures were instituted 
before performing an exploratory laparotomy 24 hours after admission. Intraoperative constant: Gas from the abdominal cavity and about $2000 \mathrm{ml}$ of a greenish liquid mixed with pus, significant deposit of fibrins on the stomach and intestinal loops, an incomplete section of the jejunum on the ante-mesenteric surface approximately $1.5 \mathrm{~cm}$ to $90 \mathrm{~cm}$ from the angle of TREITZ to the contused edges, a loosening of the gastric serosa on the front face of the stomach over a space of $2 \mathrm{~cm}$ in diameter. The surgery essentially consisted of:

- Abundant cleansing of the abdominal cavity with 5 liters of lukewarm Nacl $0.9 \%$ serum; - a freshening of the intestinal edges followed by an enterorrhaphy in separate points with Silk $2 / 0$ in a plane reinforced by sero-serous separate stitches;

- Closure of the abdominal cavity, shot by shot, after placement of two lamellar drains in the right and left colo-parietal gutters, removed 4 days later. The patient was put under; Ceftriaxone 2x1gr/d IVD, Metronidazole 3x500mg/d IVL, Paracetamol
$3 x 1 \mathrm{gr} / \mathrm{d}$ directly intravenously, Cimetidine $2 \times 200 \mathrm{mg} / \mathrm{d}$ directly intravenously. The evolution was marked by the occurrence on D6 postoperative of a fever without schedule, sweating, and hiccups. The clinical examination had objectified pale palpebral conjunctivae, tachypnea, tachycardia, disappearance of prehepatic dullness with tenderness in both flanks. An ultrasound performed on D7 showed sub-phrenic abscesses and another laparotomy was indicated and performed, and revealed two pockets of pus in the left and right sub-diaphragmatic areas. Careful adhesiolysis was done to separate the loops down to the pockets of pus, followed by aspiration, abundant washing with 5 liters of $0.9 \% \mathrm{Nacl}$ and drying of the abdominal cavity. The wall had been sutured in two planes at separate points after placement of lamellar drains, and the patient had been put on strong antibiotic therapy. The patient was discharged from the hospital on the 27th day of hospitalization cured, and seen again one month later, without particularities.
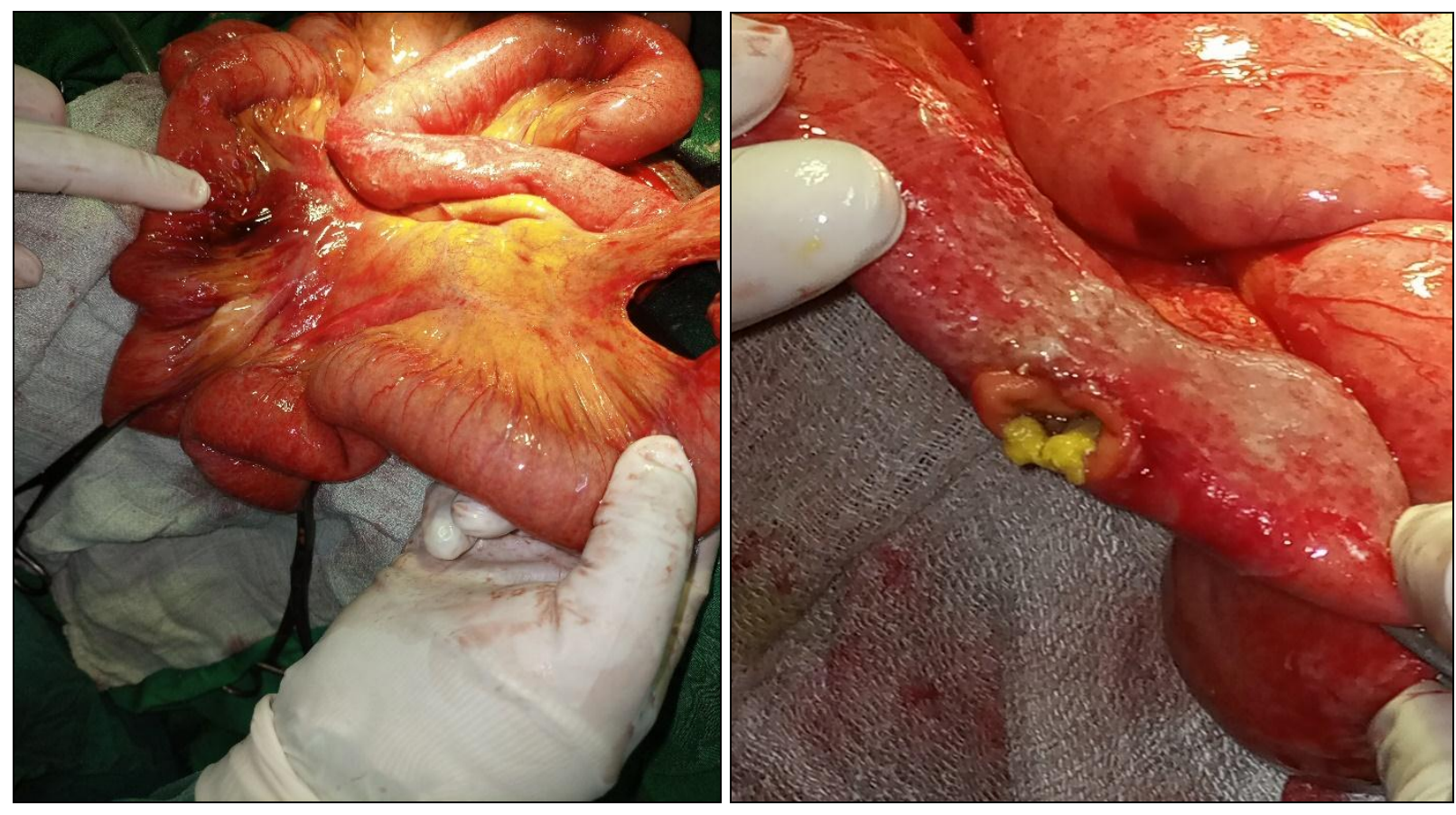

Fig 2: Images showing jejunal rupture and mesenteric tear secondary to blunt abdominal trauma.

\section{Discussion}

Traumatic digestive perforation is the pathological opening of the wall of a hollow organ of the digestive tract (esophagus, stomach, small intestine, large intestine, rectum, extra-hepatic bile ducts) following a trauma ${ }^{[6]}$. Perforation of traumatic origin of the hail can be due to a penetrating wound (stabbing, firearms), contusion of the abdomen (work and sports accident) or, exceptionally, be a complication of laparoscopy ${ }^{[7]}$. Traffic accidents are among the main causes of abdominal trauma, both in Western countries and in developing countries ${ }^{[8,9,10]}$, which corroborates with our two clinical observations all victims road traffic accident. Injuries to the small intestine during abdominal trauma are exceptional and have three essential characteristics that make the management of patients who are affected very difficult:

- Their symptoms are delayed.

- Their symptomatology may be masked due to the therapies undertaken, or on the contrary simulated due to the lesion association.

- Their ultimate consequence is generalized peritonitis, which, if it can already engage the vital prognosis in the healthy subject, seriously alters the prognosis of the polytraumatized ${ }^{[11]}$. Regarding our two observations; they had been admitted the first, 24 hours after the trauma and the second six days after the trauma, after obviously having transited in peripheral centers without adequate diagnostic and therapeutic means. This had resulted in late treatment, with a vital prognosis. Four mechanisms may be responsible for intestinal injury during abdominal contusion ${ }^{[9,12]}$ :

- Crushing between an element outside the body and the posterior plane,

- Bursts by tensioning a viscus or a segment of viscus by a sudden increase in intra-abdominal pressure,

- Stretching or tearing related to sudden deceleration phenomena, which may affect the hollow viscera such as their meso,

- Ischemia linked to meso-lesions. Given the accidental contexts for our two patients, we think that the crushing mechanism could justify the lesions we observed. The clinical signs of post-traumatic small bowel perforations are not very specific ${ }^{[13,14]}$ :

- In typical cases, the pain is constant, often very violent, but there are atypical pictures of traumatic rupture of the hail with very poor initial signs. In our two observations, 
abdominal pain was the main sign in accordance with the various data in the literature.

- Abdominal skin lesions, although non-specific, are frequent: skin erosions, bruises, related to the violence of the trauma. They must attract attention. They are sometimes related to the seat belt. Our two patients who were not wearing a seat belt during the accident therefore had no visible skin lesions; the presence or absence of cutaneous stigma therefore does not testify to the intensity of the trauma.

- The abnormal agitation of the injured person is often an evocative sign of hollow organ rupture; in opposition to this literature, our patients had no agitation probably because they came to consult late after the trauma while the picture of generalized acute peritonitis was already clear with a peritoneal facies and a classic frozen attitude in case of peritonitis.

- The very discreet abdominal defense at the start contrasts with the intensity of the pain. It progresses to contracture. Our patients not being received in the first hours, we cannot confirm this statement. However, the first patient seen 24 hours after the trauma had presented a generalized abdominal defense while the second seen 6 days after the trauma presented a picture of generalized abdominal contracture confirming that the evolution of traumatic ruptures of the small intestine is towards a table of peritonitis with abdominal defense or even a contracture. The signs of traumatic perforation of the hail are those of generalized acute peritonitis. Pain is a constant major functional sign in traumatic small bowel perforations ${ }^{[10,15]}$. The physical examination is a crucial time in these abdominal trauma patients. This clinical picture is that of a generalized acute peritonitis as confirmed by our observations. All our patients had shown signs of peritonitis. On the other hand, this generalized acute abdomen syndrome has been reported at varying rates by different authors ${ }^{[12,13]}$.

The plain abdominal X-ray can provide arguments to better establish the diagnosis by the presence of diffuse grayness and/or pneumoperitoneum (gaseous crescent) ${ }^{[7,16]}$. In doubtful cases, ultrasound and CT scan can carry the diagnosis by highlighting an intraperitoneal fluid effusion $[17,18]$. In the working conditions that are ours, and faced with the financial exhaustion of our patients, in addition to the arguments brought by the physical examination, a standard x-ray of the abdomen without preparation, and a peritoneal washing puncture (when admission of the patient is done immediately after the accident), seem to us to be efficient and help us establish the diagnosis.

In his study done in Morocco, Bellefqih Sara ${ }^{[19]}$ states that the sensitivity of peritoneal washing puncture for the diagnosis of hollow organ lesion was $100 \%$, while computed tomography was at the origin of a false negative and the ultrasound of three false negatives. PLP continues to be an important tool in the assessment of abdominal trauma. In the event of a digestive lesion, it proves to be more sensitive than ultrasound and CT scan, but it must be used in conjunction with the latter in order to reduce the rate of non-therapeutic laparotomies that it entails [19]. For our observations, our attitude was not different from those described in the literature by several authors ${ }^{[14,19]}$. The surgical act took place 48 hours after the trauma for the first and seven post-traumatic days for the second. Also, we have taken the following action: Abundant cleansing of the abdominal cavity with 5 liters of lukewarm Nacl $0.9 \%$ serum; a freshening of the intestinal edges followed by an enterorrhaphy in separate points with Silk $2 / 0$ in a plane reinforced by sero-serous separate stitches. Closure of the abdominal cavity plane by plane without suturing the peritoneum, after placement of two lamellar drains in the right and left coloparietal gutters. The patients had been put on an antibiotic combining a cephalosporin with an imidazole with also adjuvants as mentioned in the observations. In the presence of late lesions or in the presence of peritoneal contamination, as was the case for us, several authors recommend an ileostomy ${ }^{[14]}$. In our work context, this approach remains questionable because of the real difficulties imposed by the management of digestive stomas in general and particularly ileostomies; as Kibonge et al. ${ }^{[20]}$ in their studies. The evolution of our patients was marked by complications. For the first patient, a low-flow digestive fistula had appeared on the 7 th post-operative day and had been treated medically, which had dried up on the 13th day later; or on the 20th postoperative day. For the second patient, the postoperative follow-up was marked by the appearance of a clinical picture of deep suppuration with fever without schedule, abdominal pain. An ultrasound performed on the 7 th postoperative day revealed right and left subphrenic abscesses which indicated relaparotomy for drainage and abundant cleaning of the abdominal cavity. The prognosis of traumatic jejunal ruptures seems to have improved over the years, but morbidity and mortality remains relatively high in several series, varying between 3 and $12 \%{ }^{[16,17]}$. This morbidity in our case was linked to the diagnostic delay by ricochet to the delay in treatment, but we had managed to save our patients.

\section{Conclusion}

Rupture of the small intestine by blunt abdominal trauma is a rare entity. Two clinical signs are usual: sharp abdominal pain and the subsequent appearance of tusk. Abdominal skin lesions, inconstant, testify to the violence of the traumas but their absence does not exclude internal lesions. In the absence of frank peritoneal signs, clinical monitoring by careful and repeated physical examinations should be able to promote early diagnosis. We place particular emphasis on the value of the abdominal puncture-lavage which can make it possible to make the diagnosis without expensive means. The surgical treatment is simple, and their prognosis is more related to the precocity of the treatment, to the severity of the associated lesions than to the intestinal lesion.

\section{Conflicts of interest}

The authors declare no conflict of interest.

\section{Author contributions}

All authors have contributed substantially to the completion of this work.

\section{References}

1. Janzen DL, Zwirewich CV, Breen DJ, Nagy A. Diagnostic accuracy of helical CT for detection of blunt bowel and mesenteric injuries. Clin Radiol. 1998 Mar; 53(3):193-7.

2. Munshi IA, DiRocco JD, Khachi G. Isolated jejunal perforation after blunt thoracoabdominal trauma. J Emerg Med. 2006 May;30(4):393-5.

3. Essomba A. ME. Les ruptures traumatiques jejuno-ileales : a propos de dix-huit observations à l'hopital central de Yaoundé. Médecine d'Afrique Noire. 2000;47(1):4.

4. Lindenmann JM, Schmid D, Akovbiantz A. [Jejunum perforation following blunt abdominal trauma--a case report]. Schweiz Rundsch Med Prax Rev Suisse Med Prax. 1994 Aug 2 ;83(31):857-60. 
5. Suter M, KA. Les lésions de l'intestin grêle et du colon dans les traumatismes fermés de l'abdomen. MédHyg. 1992;50:69-2174.

6. Jean YM, JLC. Péritonite aiguë. Rév Prat (Paris). 2001;51:2141-45.

7. Ceelen W, Hesse U, De Hemptinne B. Small bowel perforation following blunt abdominal trauma. Acta Chir Belg. 1995;95(4 Suppl):187-9.

8. Mirvis SE, Gens DR, Shanmuganathan K. Rupture of the bowel after blunt abdominal trauma: diagnosis with CT. Am J Roentgenol. 1992 Dec; 159(6):1217-21.

9. Donohue JH, Crass RA, Trunkey DD. The management of duodenal and other small intestinal trama. World J Surg. 1985 Dec;9(6):904-13.

10. Le Kremlin. Prise en charge des ruptures d'organes creux lors des traumatismes fermes de l'abdomen Mapar2002. Flammarion éd Paris. 1998;1:234-40.

11. Bâ PA, Diop B, Soumah SA, Aidara CM, Mbaye EM, Fall B. Les lésions intestinales au cours des traumatismes fermés de l'abdomen: diagnostic et prise en charge en milieu défavorisé. J Afr Hépato-Gastroentérologie. 2013 Mar; 7(1):14-7.

12. Allen GS, Moore FA, Cox CS, Wilson JT, Cohn JM, Duke $\mathrm{JH}$. Hollow visceral injury and blunt trauma. J Trauma. 1998 Jul;45(1):69-75; discussion 75-78.

13. Hanssens F, GC Kara-Terki N, Verhaeghe P. Rupture traumatique du jéjunum non décelable par la tomodensitométrie hélicoïdale. Ann Chir. 2001;126:457486.

14. Salepcioglu H, Bertin C, Riou B. Lésion non hémorragique au cours d'un traumatisme abdominal fermé. Ann Fr Médecine Urgence. 2011 Jan;1(1):48-52.

15. Mukhopadhyay. Intestinal Injury from Blunt Abdominal Trauma: A Study of 47 Cases. Oman Med J [Internet]. 2009 [cited 2020 Apr 13]; Available from: http://www.omjournal.org/OriginalArticles/FullText/20091 0/FT_IntestinalInjuryfromBlunAbdominalTraumaAStudy.ht $\mathrm{ml}$

16. Oliveira FJ, Conçalves O, Santos JD, Martinho F, Oliveira F. [Perforations of the small intestine in closed injuries of the abdomen. Apropos of 63 cases]. J Chir (Paris). 1984 Feb; 121(2):97-100.

17. Coskun AK, Yarici M, Ulke E, Mentes O, Kozak O, Tufan T. Perforation of isolated jejunum after a blunt trauma: case report and review of the literature. Am J Emerg Med. 2007 Sep; 25(7):862.e1-862.e4.

18. Bellefqih Sara. Traumatismes abdominaux: La Ponctionlavage du péritoine a-t-elle encore une place? (A propos de 90 cas). FMPR. Theses de médecine. 2009 Dec $16 ;(226): 122$.

19. Pouget X, Mallet T, Kalfon M, Descottes B. [Wounds of the small intestine in closed injuries of the abdomen. Apropos of 14 cases]. Ann Chir. 1983 Sep;37(6):389-92.

20. Kibonge A M, Bisimwa N M., Bagale Y B., Kanganda DMK., Munyantwari E. A, Tshimbila JMV. K. Indications et complications des stomies digestives en millieux sousdévelopés. Revue Médical des Grands Lacs. 2019;10(1):4751. 